\title{
Aktuelle Information des DZK zur Tuberkulinversorgung Deutschlands
}

R. Loddenkemper

\section{Supply of Tuberculin in Germany}

Der Hersteller des einzigen bisher in Deutschland für Intradermaltests nach Mendel-Mantoux verwendeten Tuberkulins, die Firma Chiron Vaccines Behring, hat im vergangenen Jahr überraschend die Produktion von Tuberkulin Behring GT eingestellt. Derzeit werden nur noch Restbestände vertrieben. Als Grund für die Produktionseinstellung wurde die Erneuerung des Werks in Marburg angeführt. Eine Modernisierung der Tuberkulinproduktion hätte sich nach Einschätzung des Herstellers nicht mehr gelohnt.

Im November 2004 fand zu diesem Thema in Berlin ein Treffen von Vertretern von Chiron Vaccines Behring, des Deutschen Zentralkomitees zur Bekämpfung der Tuberkulose (DZK), des Robert Koch-Instituts (RKI) und des Öffentlichen Gesundheitsdienstes statt. Nach Aussage der Vertreter Chiron Vaccines Behring gingen eigene Berechnungen davon aus, dass die Bestände an GT 10 den Bedarf bis zum April des Jahres 2005 decken würden. Die Zuverlässigkeit dieser Berechnungen musste allerdings aufgrund der Umstellung vom Stempeltestverfahren, dessen Produktion bereits 2003 ausgelaufen war, auf die Testung nach Mendel-Mantoux hinterfragt werden. Mittlerweile sind die Bestände an GT 10 bereits erschöpft, geringe Restbestände existieren derzeit noch für die Dosierungen GT 100 und GT 1000.

Als Übergangslösung bietet Chiron Vaccines Behring den Import des von Chiron S.r.l. in Siena hergestellten italienischen Tuberkulins Biocine PPD 5 IE lyophil an, welches über eine in Deutschland ansässige Firma bezogen werden kann (weitere Informationen unter http://www.chiron-behring.de und http://www.chiron-behring.de/cont_184.php?news_id=487).
Da es sich um ein in Deutschland nicht zugelassenes Produkt handelt, ist die Einzelverordnung nach $\S 73$ Abs. 3 Arzneimittelgesetz notwendig. Der Verkauf des Biocine-Test PPD lyophil erfolgt ebenfalls bereits aus Restbeständen, da auch das italienische Werk in Siena die Produktion schon eingestellt hat. Die Tuberkulin-Versorgung Italiens und Deutschlands soll nach Aussage von Chiron Vaccines Behring jedoch bis 2008 gewährleistet sein.

Langfristig will Chiron Vaccines Behring die Versorgung Deutschlands und Italiens durch in Großbritannien (Liverpool) produziertes Tuberkulin (PPD Evans) sicherstellen. Auch das dortige Werk wird zurzeit modernisiert, die Produktion soll im April des Jahres 2006 wieder aufgenommen werden (Lieferbeginn voraussichtlich ab Mai 2006).

Bioäquivalenzstudien für die genannten Tuberkuline liegen nach Auskunft von Chiron Vaccines Behring nicht vor.

Als weitere Möglichkeit können, neben den erwähnten Tuberkulinen der Firma Chiron Vaccines, auch das von der Weltgesundheitsorganisation (WHO) als Standardtuberkulin empfohlene und in vielen europäischen Ländern bereits eingeführte Tuberkulin PPD RT23 des Statens Serum Institut (Kopenhagen, Dänemark; www.ssi.dk/tuberculin) sowie das in den USA verwendete Tubersol (PPD CT68) importiert werden.

Es wird angenommen, dass die Tuberkuline folgender Dosen äquivalent sind: 10 TE Behring, 5 TU PPD-S (USA), 2 TU RT23-SSI, 5 IE Biocine PPD und wahrscheinlich auch $10 \mathrm{U}$ Evans PPD. 
Zusammen mit dem RKI und dem Paul-Ehrlich-Institut (PEI) bemüht sich das DZK intensiv um eine Problemlösung, da eine kontinuierliche Tuberkulin-Versorgung Deutschlands zu diagnostischen Zwecken und im Rahmen von Umgebungsuntersuchungen zwingend notwendig ist. Angestrebtes Ziel sollte zudem die Verwendung eines einheitlichen Tuberkulins in allen Regionen sein, da ansonsten ein standardisiertes Vorgehen und die Vergleichbarkeit auf nationaler Ebene gefährdet sind.

Für das zukünftig in Deutschland verwendete Tuberkulin sollten folgende Anforderungen erfüllt sein:

- Bioäquivalenz zum internationalen Referenz-Tuberkulin PPD-S (Seibert) zur Gewährleistung einer standardisierten Dosis und standardisierten Interpretation der Testergebnisse ${ }^{1}$

- Gute Datenlage/umfangreiche Produkterfahrung

- Zulassung für Deutschland

- Verlässliche und unkomplizierte Liefer- und Verfügbarkeit

- Möglichst verbraucherfreundliche Produkteigenschaften(Anwendungszeiträume, Lagerung, verfügbare Dosen)

- Möglichst geringe Rate an Starkreaktionen

- Möglichst geringe Kreuzreagibilität mit nicht-tuberkulösen Mykobakterien

- Akzeptables Preis - Leistungsverhältnis

Um den derzeitigen Engpass in der Tuberkulin-Versorgung zu überbrücken, ist der Import leider unumgänglich. Das PEI informiert zum aktuellen Stand und zum Bezug von Arzneimitteln gemäß $§ 73$ Abs. 3 des Arzneimittelgesetzes auf seiner Homepage (http:/www.pei.de/professionals/tuberkulinversorgung.htm).

Zur Frage der Haftung der Ärzte im öffentlichen Gesundheitsdienst im Falle der Anwendung eines in Deutschland nicht zugelassenen Produktes nahm das Sozialministerium des Landes Baden-Württemberg am 11.2.2005 in einem Schreiben an die Land- ratsämter bzw. Bürgermeisterämter folgendermaßen Stellung: „Bei hoheitlicher Tätigkeit des Arztes - z. B. bei Aufgaben des öffentlichen Gesundheitsdienstes - haftet grundsätzlich der Staat bzw. die Körperschaft, in deren Dienst der Arzt steht. Eine persönliche Haftung des verschreibenden Arztes des Gesundheitsamts kommt - in Form eines Rückgriffs - nur bei Vorsatz und grober Fahrlässigkeit in Betracht (Art. 34 Satz 2 GG). Bei der Verschreibung von Einzeleinfuhrpräparaten nach §73 Abs.3 AMG richtet sich die Verantwortlichkeit des Arztes bei Pflichtverletzungen nach den allgemeinen Grundsätzen des Schadensersatzrechts. Je nachdem, in welchem Land das Arzneimittel zugelassen ist, sind dabei höhere Sorgfalts- und Prüfpflichten bei der Verschreibung zu beachten. Bei einem in einem EU-Mitgliedsstaat zugelassenen Arzneimittel dürften insoweit allerdings keine besonderen Anforderungen bestehen."

Das Statens Serum Institut prüft derzeit die Möglichkeit einer Zulassung des PPD RT 2359 SSI in Deutschland. Chiron Vaccines Behring strebt keine Zulassung für das Biocine-Tuberkulin an, hat aber die Absicht, eine Zulassung für das Evans-Tuberkulin in Deutschland zu beantragen. Bis in Deutschland wieder ein zugelassenes Tuberkulin zur Verfügung steht, wäre es, insbesondere in Fällen, wo ein Lagerbestand an Tuberkulin unverzichtbar ist, wie beispielsweise im Öffentlichen Gesundheitsdienst und in Krankenhausapotheken, überaus hilfreich, wenn eine Ausnahmeregelung für den Import unter Umgehung des $§ 73$ Abs. 3 Arzneimittelgesetz erwirkt werden könnte.

Bei einem beschleunigten Zulassungsverfahren unter Einbeziehung des PEI und der dänischen Behörden könnte die Zulassung in Deutschland in 2-3 Monaten erfolgen. Es wird weiter intensiv daran gearbeitet, baldmöglichst in Deutschland wieder ein zugelassenes und qualitativ hochwertiges Tuberkulin zur Verfügung zu haben.

\footnotetext{
${ }^{1}$ Optimal wäre eine nachgewiesene Bioäquivalenz zum bisher verwendeten Tuberkulin, um die bisherigen Erfahrungen in der Interpretation ohne größere Schwierigkeiten übertragen zu können.
} 